\title{
V. Ueber eine neue klinische Methode zur quantitativen Bestimmung von Quecksilber im Harn und die Ausscheidung dieses Metalles bei mit löslichem metallischem Quecksilber behandelten Kranken.

\author{
Von Dr. Friedrich Eschbaum,
}

Vorsteher der Apotheke der Kgl. thieräratlichen Hochschule in Berlin.

Bei dem grossen Interesse, das den wasserlöslichen Metallen seit ihrer Entdeckung seitens der Chemiker und nicht minder seitens der Aerzte zugewendet wird, habe ich es für angezeigt gehalten, die Ausscheidung dieser Metalle und zwar zunächst die des Quecksilbers durch den Harn zu studieren.

Die zur quantitativen Bestimmung des Quecksilbers im Harn vorhandenen Methoden sind so mühsam, zeitraubend und kostspielig, dass sie nur von wenigen Forschern angewendet worden sind. Wie wenig sie sich aber zu klinischen Untersuchungen eignen, zeigen die Arbeiten von E. Welander, des auf diesem Gebiete hervorragenden Forschers, der wiederholt den Werth der quantitativen Bestimmung des Quecksilbers im Harn hervorgehoben hat, aber trotzdem eine einfache Methode der approximativen Bestimmung denen der quantitativen vorzieht.

Die zumeist angewendete quantitative Methode ist die von Winternitz 1 : das Quecksilber wird nach einer modificirten

1) Winternitz, Archiv für experimentelle Pathologie und Pharmakologie 1889. 
Amalgamationsmethode aus dem Harn gewonnen und in eine Vorlage sublimirt.

Léon Brasse ${ }^{1}$ ) fängt die Dämpfe des Quecksilbers, welches er nach der Amalgamationsmethode aus dem Harn gewonnen hat, auf einem Golddeckel auf.

A. Jolles ${ }^{2}$ ) reducirt durch Zinnchlorür das Quecksilber im Harn und schlägt es auf Goldkörner nieder.

Endlich hat man es durch Elektrolyse zu bestimmen gesucht. Die Methoden, deren sich der Chemiker zur quantitativen Bestimmung des Quecksilbers bedient, kommen bei den geringen Mengen des Metalles, wie sie sich inı Harn finden, kaum in Betracht.

Eine für vorliegende $Z$ wecke brauchbare Methode muss einfach sein, da jede Complication die Schärfe der Reaction trübt und ihre Brauchbarkeit für die Praxis in Frage stellt.

Ich binde nach der ïblichen Methode mit einigen Cautelen das im Harn befindliche Quecksilber an Kupfer, trenne es von letzterem durch Erhitzen und nehme es dann mit einem Stückchen metallischen Silbers von den Wandungen des Reagensglases weg. Durch Wägung des Silberplättchens vor und nach der Amalgamirung findet man die Menge des vorhandenen Quecksilbers.

Der zu untersuchende Harn wird mit etwas Traubenzucker versetzt und erhitzt. Während des 15 Minuten langen Kochens setzt man in kleinen Antheilen eine zur Ausfällung der Phosphate ausreichende Menge Natronlauge (etwa $15 \mathrm{ccm}$ pro Liter) zu, lässt absetzen, giesst die über dem Niederschlag stehende Flüssigkeit ab und bringt den Rückstand in ein grösseres Reagensglas. Nachdem er sich wiederum gesetzt hat, trennt man ihn nochmals durch Abgiessen von der Flüssigkeit und giebt nun etwa ein Zehntel seines Volums reiner officineller Salzsäure hinzı, mit der man vorher den grossen Kolben ausgespült hat. Nun giebt man ein der Höhe und dem Dickendurchmesser der Flüssigleit entsprechendes Stück Kupferdrahtnetz ${ }^{3}$ ) hinzu und stellt das Glas so in ein Wasserbad, dass es bis über seinen Inhalt von siedendem Wasser umgeben ist, und lässt es so etwa $1 / 3$ Stunde kochen. Nun wird die Flamme unter dem Wasserbade entfernt und das Glas erst lose, nachher fest verschlossen mindestens 24 Stunden im Wasserbade stehen gelassen. Letzteres soll nicht ganz erkalten, sondern auf 45 bis $60^{\circ}$ erwärmt bleiben. Nun wird die Flüssigkeit abgegossen, das Kupfernetz erst mit Wasser, dann mit Wasser, dem man etwas Natronlauge zugefügt hat, und schliesslich wieder mit reinem, am besten lauwarmem Wasser gründlich gewaschen. Um es zu trocknen, wäscht man es erst mit Alkohol, dann mit Aether und lässt es eine halbe Stunde an der Luft liegen ${ }^{4}$ ). Es wird dann in ein gewöhnliches Reagensglas mit Hülfe eines Glasstabes fest eingedriickt. Der untere Theil des Glases, in dem sich das zusammengedrückte Kupfernetz befindet, wird mit einem Bunsenbrenner stark erhitzt: Das Quecksilber sublimirt und sammelt sich über dem Kupfer an den Wandungen des Glases an. In dieses Reagensglas, an dessen Boden das Kupfernetz sitzt, bringt man ein etwa $21 / 2 \mathrm{~cm}$ langes, $0,7 \mathrm{~cm}$ breites, $1 \mathrm{~mm}$ dickes Silberplättchen und dreht das in wagerechter Lage befindliche Reagensglas so, dass das Silberplättchen an den Wandungen, an denen das Quecksilber sitzt, entlang gleitet. Das Drehen, oder auch vorsichtiges Schïtteln ist solange fortzusetzen, bis man beim Hineinsehen durch die obere Oeffnung (bei auffallendem Licht) keinen Beleg mehr wahrnehmen kann.

Das Quecksilber amalgamirt sich sofort mit dem Silber; nach ein, höchstens zwei Minuten langem Drehen, bezw. Schütteln ist alles Quecksilber vom Silber aufgenommen. Die Gewichtszunahme des Silberplättchens giebt die Menge des vorhandenen Quecksilbers an. lch nehme die letztere Operation, die somit höchstens zwei Minuten in Anspruch nimmt, an der chemischen. Wage sitzend vor: es liegt zwischen der Wägung des Silberplättchens und der des mit

1) Léon Brass e, Compt. rend. soc. biolog. 1887.

2) A. Jolles, Monatshefte für Chemie 16. Wiener medicinische Presse 1895

3) Das Kupferdrahtnetz muss ein Geflecht aus feinem Draht sein, wie es zu Spiralen in der organischen Analyse verwendet wird. Es eignet sich nur reines Kupfer: zinkhaltiges ist für diese Reaction nicht zu gebrauchen, also auch nicht Lametta.

*) Bisweilen kommt es vor, dass das Kupfernetz von darauf niedergeschlagener organischer Substanz aus dem Harn dunkelbraun bis schwarz erscheint. In diesem Falle lässt man es mit Wasser, dem man etwas Natronlauge zugesetzt hat, so lange stehen, bis es metallisch oder doch rein gelb geworden ist.
Quecksilber beladenen also eine nur sehr kurze Zeit. Dies ist aber gerade für das Gelingen der Wägung von so minimalen Mengen von ausserordentlichem Werth, weil nur auf diese Weise die Wägungsfehler soweit vermieden werden, wie es überhaupt möglich ist.

Das Silberplättchen wird auf einem Uhrglas auf die Wage gebracht. Es wird vor dem Gebrauch durch Erhitzen von etwa von einer früheren Operation herrührendem Quecksilber befreit und mit einem Stückchen Schmirgel- oder Glaspapier glänzend geputzt.

Es ist zweckmässig, unmittelbar nach jeder Bestimmung das Silberplättchen durch Erhitzen in einem Reagensglas vom Quecksilber zu befreien; man kann dann in dem Glas, nachdem man es abgesprengt und das Silberplättchen von unten entfernt hat, die Jodprobe in der bekannten Weise führen. ${ }^{1}$ )

Bei nicht allzu geringen Mengen von Quecksilber kann man es auch aus dem Gewichtsverlust bestimmen, den das mit Quecksilber amalgamirte Silberplättchen beim Erhitzen erfährt. Diese indirekte Bestimmung hat fïr denjenigen Arzt, dem keine chemische Wage zur Verfügung steht, den Vortheil, dass er das präparirte, mit Quecksilber beladene Silberplättchen einem Chemiker zur

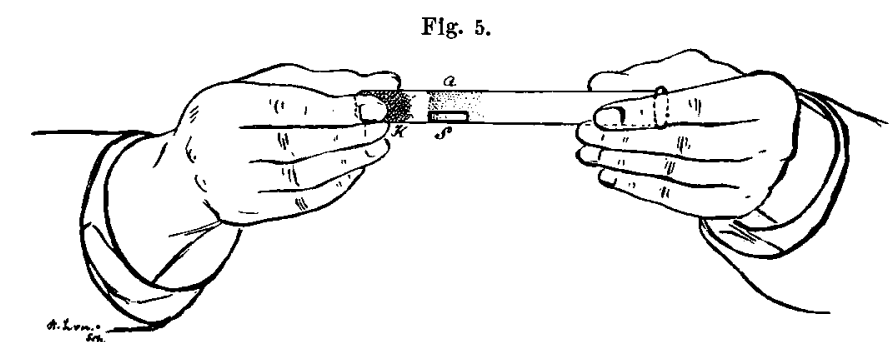

$S=$ Silberplättchen; $K=$ zusammengedrïcktes Kupferdrahtnetz; $Q=$ Quecksilberbelag.

Wägung übermitteln kann. Der letztere wägt das Silberplättchen sammt anhaftendem Quecksilber, erhitzt es dann in einem Reagensglase vorsichtig, aber ziemlich stark, sprengt den Boden des letzteren $a b$, nimmt das Silberplättchen von unten heraus und wägt es nach dem Erkalten wiederum.

Als Beläge für die Brauchbarkeit der Methode führe ich folgende Versuche an:

a) Versuche mit reinem, metallischem Quecksilber,

An den Boden eines Reagensglases wurde ein minimales Quecksilberkiigelchen gebracht; es wog 0,0017. Der Boden des Glases wurde erhitzt und das sublimirte Quecksilber mit einem Silberplättchen in der vorher beschriebenen Weise amalgamirt; es hatte an Gewicht zugenommen 0,0016. halten;

In derselben Weise verfahren wurden folgende Zahlen er-

$$
\begin{aligned}
& \text { abgewogen 0,0036 Quecksilber, gefunden 0,00356, } \\
& 0,0029 \quad \cdots \quad, 0,00284 \text {, } \\
& 0,0026 \quad " \quad . \quad \quad \quad \quad \quad 0,0026 \text {. }
\end{aligned}
$$

b) Versuche mit wässeriger Quecksilberchloridlösung. ${ }^{2}$ )

0,00542 Quecksilberchlorid $=0,0040$ Quecksilber wurden mit $200 \mathrm{ccm}$ Wasser und $20 \mathrm{ccm}$ reiner officineller Salzsäure und einem etwa $12 \mathrm{~cm}$ langen und $1,4 \mathrm{~cm}$ breiten Stück Kupferdrahtnetz erst im siedenden, dann im allmählich erkaltenden Wasserbade bei $45-60^{\circ} 24$ Stunden behandelt. Das aus dem Kupfer durch Sublimation erhaltene und an Silber gebundene Quecksilber betrug 0,0038 .

In derselben Weise wurden folgende Zahlen erhalten:

0,00813 Quecksilberchlorid = $\mathbf{0 , 0 0 6 0}$ Quecksilber, $220 \mathrm{ccm}$ Wasser, $20 \mathrm{ccm}$ Salzsäure, gefunden $\mathbf{0 , 0 0 5 8 5} \mathrm{Hg}$. 0,00271 Quecksilberchlorid $=\mathbf{0 , 0 0 2 0}$ Quecksilber, $200 \mathrm{ccm}$ Wasser, $20 \mathrm{ccm}$ Salzsäure, gefunden $\mathbf{0 , 0 0 1 7} \mathrm{Hg}$.

1) Diese Operation ist dann unerlässlich, wenn die Quecksilbermenge so gering ist, dass sie keinen Ausschlag auf der Wage hervorruft; man kann dann wenigstens den qualitativen Nachweis führen.

2) $\mathrm{Zu}$ dieser Versuchsreihe wurde eine Lösung verwendet, die im Liter 0,1355 Qnecksilberchlorid enthält =0,1 Quecksilber. $1 \mathrm{ccm}$ dieser Lösung enthält also $1 / 10 \mathrm{mg}$ Quecksilber. Für den ersten Versuch wurden hiervon $40 \mathrm{ccm}=4 \mathrm{mg}$ Quecksilber, für den zweiten $60 \mathrm{ccm}=$ $6 \mathrm{mg} \mathrm{Hg}$, für den dritten $20 \mathrm{ccm}=2 \mathrm{mg}$ Quecksilber abgemessen u. s. w. 
0,00542 Quecksilberchlorid $=\mathbf{0 , 0 0 4 0}$ Quecksilber,

$220 \mathrm{ccm}$ Wasser, $20 \mathrm{ccm}$ Salzsäure, gefunden $\mathbf{0 , 0 0 3 4 5 ~ H g}$.

0,001355 Quecksilberchlorid $=\mathbf{0 , 0 0 1 0}$ Quecksilber,

$200 \mathrm{ccm}$ Wasser, $20 \mathrm{ccm}$ Salzsäure, gefunden $0,0007 \mathrm{Hg}$.

c) Versuche mit Harn, dem Quecksilberchlorid zugesetzt w urde. $\left.{ }^{1}\right)$

$200 \mathrm{ccm}$ Harn, 0,00813 Quecksilberchlorid = 0,0060 Quecksilber, $20 \mathrm{ccm}$ Salzsäure, $60 \mathrm{ccm}$ Wasser, gefunden $\mathbf{0 , 0 0 5 6 2} \mathrm{Hg}$.

$200 \mathrm{ccm}$ Harn, 0,00542 Quecksilberchlorid = 0,0040 Quecksilber, $20 \mathrm{ccm}$ Salzsäure, $40 \mathrm{ccm}$ Wasser, gefunden $\mathbf{0 , 0 0 2 7 5} \mathrm{Hg}$.

$200 \mathrm{ccm}$ Harn, 0,00271 Quecksilberchlorid = 0,0020 Quecksilber, $20 \mathrm{ccm}$ Salzsäure, $20 \mathrm{ccm}$ Wasser, gefunden $\mathbf{0 , 0 0 1 5} \mathrm{Hg}$.

Bei diesen Versuchen wurde der oxydirende Einfluss der Luft durch ein auf den Kolben gesetztes Gummischlauchventil verhindert. Auch habe ich das verwendete Kupfernetz vor dem Gebrauch durch Reduction mittels Methylalkohols (Erhitzen des Kupfernetzes in der Flamme und Einlegen des schwach glühenden Metalls in ein weites Reagensglas, dessen Wandungen mit Methylalkohol befeuchtet sind, und sofortiges Schliessen des Glases mit einem Korkstopfen) vom Oxyd befreit. Ich bemerke dies bloss, um meine Versuche so $\mathrm{zu}$ beschreiben, wie sie angestellt worden sind. Zum Gelingen des Versuchs ist weder die Absperrung der Luft durch das Ventil, noch das Metallischmachen des Kupfernetzes erforderlich.

Diese Resultate sind mit der Theorie fast übereinstimmende; verdünnt man aber die Lösungen, deren Volum in den bisherigen Versuchen $250 \mathrm{ccm}$ nicht ïberschritten hat, weiter, so fallen die Zahlen wesentlich ungünstiger aus:

$0,00271 \mathrm{HgCl}_{2}=\mathbf{0 , 0 0 2 0} \mathrm{Hg}, 500 \mathrm{ccm}$ Wasser, $20 \mathrm{ccm}$ Salzsäure, gefunden $\mathbf{0 , 0 0 1 1 5} \mathrm{Hg}$.

$0,00542 \mathrm{HgCl}_{\mathbf{2}}=\mathbf{0 , 0 0 4 0} \mathrm{Hg}, 500 \mathrm{ccm}$ Wasser, $20 \mathrm{ccm}$ Salzsäure, gefunden $0,0027 \mathrm{Hg}$.

$0,00542 \mathrm{Hg} \mathrm{Cl}_{2}=\mathbf{0 , 0 0 4 0} \mathrm{Hg}, 900 \mathrm{ccm}$ Wasser, $20 \mathrm{ccm}$ Salzsäure, gefunden $\mathbf{0 , 0 0 1 4} \mathrm{Hg}$.

$0,00542 \mathrm{HgCl}_{\mathbf{2}}=\mathbf{0 , 0 0 4 0} \mathrm{Hg}, 900 \mathrm{ccm}$ Wasser, $100 \mathrm{ccm}$ Salzsäure, gefunden $0,0017 \mathrm{Hg}$

Aus dieser Versuchsreihe geht hervor, dass eine gewisse Concentration vorhanden sein muss, um eine quantitative Ausscheidung des Quecksilbers aus wässeriger Lösung durch Kupfer zu bewirken. Deswegen habe ich auch das Schî̉llberg-Welander'sche ${ }^{2}$ ) Concentrationsverfahren für die Untersuchung des Harnes vorgeschlagen und in den nachfolgenden Versuchen mit luetischen Harnen durchgeführt, gleichwohl ich mir bewusst bin, dass auch dadurch nicht alles Quecksilber gewonnen wird, meine Methode somit keine absoluten Werthe liefert. Die Zahlen aber geben ein objectives Bild über die Quecksilberelimination. Gleichwohl werde ich durch Versuche eine tabellarische Zusammenstellung ausarbeiten, wonach diese relativen Zahlen in absolute Werthe umzurechnen sind.

Auf die unvollständige Ausscheidung des Quecksilbers aus dem Harn mittels der Amalgamationsmethoden ist von verschiedenen Autoren hingewiesen, und zahlreiche Vorschläge, sie zu verbessern, sind gemacht worden. Ich erwähne nur die Arbeiten von Ludwig, Fürbringer, F. Müller, Alt, Almén, Paschlis, Lehmann, Schridde, besonders von Wolff und Nega und anderen Autoren, ohne übrigens näher darauf einzugehen, da es mir bei dieser Arbeit lediglich darauf ankam, das nach dem Amalgamationsverfahren aus dem Harn erhaltene Quecksilber quantitativ zu bestimmen.

Ich berichte nun über die Untersuchung des Harnes luetischer Patienten, die mit dem von Dr. 0 . Werler in die Therapie eingeführten $10 \%$ Unguentum Hydrargyri colloidalis ${ }^{3}$ ) in dessen Poliklinik behandelt worden sind.

Das Versuchsmaterial war als Prüfstein für meine Methode nicht gerade günstig, denn diese Harne sind so arm an Quecksilber, dass der

1) Auch zu dieser Versuchsreihe wurde die oben beschriebene Quecksilberchloridlösung verwendet. Der Harn wurde direkt mit Salzsäure und Kupferdrahtnetz, also ohne ihn erst auszufällen, behandelt.

2) E. Welander, Recherches sur l'absorption et sur l'élimination du mercure dans l'organisme humain. Annal. de derm. et de syphil. Tom VII, p. 412.

3) 0 . Werler, Ueber Anwendungsweise und Wirkungen des löslichen, metallischen Quecksilbers, Berliner klinische Wochenschrift 1898, No. 42, und Das lösliche metallische Quecksilber als Heilmittel, Dermatologische Zeitschrift Bd. VI, 3. Heft.
Versuch, es quantitativ zu bestimmen, nur bei einem grösseren Quantum, bei zwei Litern, gelungen ist. Dass ich nicht allzu viele Zahlen angeben kann, beruht auf der Schwierigkeit der Beschaffung von Harn in solchen Quantitäten von ambulant behandelten Patienten. Zur Feststellung der kleinen Gewichtsmengen habe ich mich der Wägungsmethode der Schwingungsbeobachtungen bedient, die u. a. Prof. Blasius ${ }^{1}$ ) beschreibt. Solche minimalen Gewichtsmengen, die man vielfach als unwägbar bezeichnet, lassen sich nach meiner Methode noch mit Sicherheit ermitteln, aus Gründen, die ich oben erörtert habe. Im Harn von Patienten, die mit der gewöhnlichen grauen Salbe klinisch behandelt waren, habe ich auch nicht wesentlich grössere Quantitäten Quecksilber gefunden, dahingegen konnte ich es schon in kleinen Mengen Harn von mit Sublimateinspritzungen behandelten Kranken stets quantitativ bestimmen.

Der Harn wurde in der oben beschriebenen Weise behandelt. Das Kupferdrahtnetz wurde vor jedem Gebrauch reducirt, ein Verfahren, das nicht mehr Arbeit macht, als einfaches Ausglïhen, was ja vor jeder Operation erforderlich ist. Die verwendeten Reagentien, Lauge und Salzsäıre, hatten sich durch den Versuch als frei von Quecksilber erwiesen. Die Patienten, deren Harn ich untersuchte, wurden an den Geschlechtstheilen nicht lokal mit quecksilberhaltigen Arzneien behandelt; sie hatten die Anweisung, nach dem Einreiben die Hände gründlich zu säubern, um eine Uebertragung von Quecksilber in den Harn zu vermeiden. Auch wurden sie nicht mit jodhaltigen Arzneien behandelt.

Fall 1. Frl. J. T., 41 Jahre alt. Erste Cur. Frische syphilitische Infection. Patientin von schwächlicher Constitution, gleichzeitig an hochgradiger Kyphose leidend, bekommt täglich 1,5 $\mathrm{g}$ Unguentum $\mathrm{Hy}-$ drargyri colloidalis zur Einreibung und hat an 53 Tagen im ganzen $80 \mathrm{~g}$ Salbe $=8 \mathrm{~g}$. Hydrargyrum colloidale verbraucht.

Harnuntersuchung: 2 Liter Urin, specifisches Gewicht 1,020, gefunden $0,11 \mathrm{mg} \mathrm{Hg}$.

Fall 2. Herr M. S., 23 Jahre alt. Dritte Cur, ein halbes Jahr nach der zweiten begonnen. An 50 Tagen je $2,0 \mathrm{~g}$ Unguentum Hydrargyri colloidalis eingerieben, mithin eine Gesammtdosis von $100 \mathrm{~g}$ Salbe $=10 \mathrm{~g}$ Hydrargyrum colloidale verschmiert.

Harnuntersuchung: 2 Liter Harn, specifisches Gewicht 1,018, gefunden $0,05 \mathrm{mg} \mathrm{Hg}$.

Fall 3. Frl. B. M., 26 Jahre alt. Erste Cur. Induratives Oedem des rechten Labium majus. Psoriasis syphilitica. An 70 Tagen wurden je $2 \mathrm{~g}$ Salbe, im ganzen $140 \mathrm{~g}$ Salbe $=14 \mathrm{~g}$ colloidales Quecksilber eingerieben.

Harnuntersuchung: 2 Liter Harn, Specifisches Gewicht 1,021, gefund en $0,12 \mathrm{mg} \mathrm{Hg}$.

Fall 4. Frl. E. D., 21 Jahre alt. Erste Cur. Ulceröses Syphilid des linken Nasenflügels, sowie luetisches Geschwür am rechten Mundwinkel. An 20 Tagen wurden bisher zusammen $40 \mathrm{~g}$ Salbe $=4 \mathrm{~g}$ Hydrargyrum colloidale eingerieben.

Harnuntersuchung: 2 Liter Harn, specifisches Gewicht 1,020, gef und en $0,06 \mathrm{mg} \cdot \mathrm{Hg}$.

Fall 5. Herr L. H., 25 Jahre alt. Erste Cur. Frische syphilitische Infection. Patient hat vor 14 Tagen die Cur ausgesetzt und am Tage, bevor er den Urin Sammelte, mit neuen Einreibungen begonnen. Gesammtdosis der eingeriebenen Salbe $146 \mathrm{~g}=14,6 \mathrm{~g}$ Hydrargyrum colloidale.

Harnuntersuchung: 2 Liter Harn, specifisches Gewicht 1,020, gefunden $0,27 \mathrm{mg} \mathrm{Hg}$.

Fall 6. Herr F. H., 19 Jahre alt. Zweite Cur. Recidiv in Form einer Syphilis cutanea papulo-Squamosa. An 35 Tagen wurden zusarnmen $70 \mathrm{~g}$ salbe $=7 \mathrm{~g}$ Hydrargyrum colloidale eingerieben.

Harnuntersuchung: 2 Liter Harn, specifisches Gewicht 1,019, gef unden $0,16 \mathrm{mg}$ : $\mathrm{Hg}$.

Fall 7. Herr H. S., 31 Jahre alt. Dritte Cur. Grosspapulöses Syphilid. Recidiv. Hat am Abend vorher die Schmiercur mit $2 \mathrm{~g}$ Unguentum Hydrargyri colloidalis begonnen. Zur Untersuchung gelangten $950 \mathrm{ccm}$ Harn, der Quecksilberbefund war negativ.

Nach der zweiten Einreibung mit je 2 g Salbe wurden untersucht $640 \mathrm{ccm}$ Harn, specifisches Gewicht 1,019. Positiver qualitativer Quecksilbernachweis.

Nach der dritten Einreibung mit 2 g Salbe wurden untersucht $510 \mathrm{ccm}$ Harn, specifisches Gewicht 1,019. Positiver qualitativer Quecksilberbefund.

Fall 8. Frl. M. O., 24 Jahre alt. Dritte Cur. Psoriasis palmaris syphilitica, Condylome an der Nase, hat vor sechs Wochen die Schmiercur mit $120 \mathrm{~g}$ Salbe beendigt.

Harnuntersuchung: 2 Liter Harn, specifisches Gewicht 1,021, gef unden $0,22 \mathrm{mg} \mathrm{Hg}$.

Fall 9. Frl. W. S., 23 Jahre alt. Zweite Cur. Syphilitische Geschwüre der Zungen- und Wangenschleimhaut, hat jeden zweiten Tag $1 \mathrm{~g}$ Salbe, im ganzen $120 \mathrm{~g}=12 \mathrm{~g}$ colloides Quecksilber verschmiert. Harnuntersuchung: 2 Liter Harn, specifisches Gewicht 1,020,

1) E. Blasius, Physikalische Uebungen. Leipzig 1895. 
hat keine Zahl, aber qualitativen Quecksilbernachweis ergeben.

Fall 10. Frl. L. W., 25 Jahre alt. Zweite Cur. Recidiv. Papulöses Syphilid im Gesicht. Hat vor acht Tagen die Schmiercur mit $180 \mathrm{~g}$ Salbe beendigt.

Harnuntersuchung: 2 Liter Harn, specifisches Gewicht 1,020. gefunden $0,15 \mathrm{mg} \mathrm{Hg}$.

Ausser diesen quantitativen Bestimmungen wurden noch 23 Harne, die nur in kleineren Quantitäten zur Verfügung standen, qualitativ untersucht. In vier Fallen ist der Quecksilbernachweis negativ, in 19 positiv ausgefallen.

Aus diesen Versuchen geht hervor, dass nach der Inunction mit dem von Werler angegebenen Unguentum Hydrargyri colloidalis, analog den übrigen Applicationsmethoden des Quecksilbers, bereits 48 stunden nach der ersten Einreibung Quecksilber im Harn erscheint und dauernd in wägbaren Mengen darin nachgewiesen werden kann, dass es noch sechs Wochen nach beendigter Cur - Harne von Patienten, bei denen die Beendigung der Cur noch weiter zuriickliegt, standen nicht zur Verfügung - durch die Nieren eliminirt wird.

Bei diesen und sehr vielen anderen, hier nicht angeführten quantitativen Quecksilberbestimmungen habe ich es fast nie unterlassen, die Zuverlässigkeit meiner Methode mittels der Jodprobe zu controlliren. Sie wurde in dem Reagensglas, nachdem der Quecksilberbelag durch das Silberplättchen weggenommen und das Kupferdrahtnetz nach Absprengung des Glases entfernt worden war, angestellt: in den meisten Fällen habe ich keine, in wenigen eine so schwache Jodidreaction erhalten, dass es nur einem durch die Uebung geschärften Auge gelingt, sie zu entdecken: ein Beweis, dass meine Methode zuverlässig ist. 